\title{
Identification of interneurons required for the aversive response of Caenorhabditis elegans to graphene oxide
}

\author{
Guosheng Xiao ${ }^{1}$, He Chen², Natalia Krasteva ${ }^{3}$, Qizhan Liư and Dayong Wang ${ }^{2 *}$
}

\begin{abstract}
Background: So far, how the animals evade the environmental nanomaterials is still largely unclear. In this study, we employed in vivo assay system of Caenorhabditis elegans to investigate the aversive behavior of nematodes to graphene oxide (GO) and the underlying neuronal basis.

Results: In this assay model, we detected the significant aversive behavior of nematodes to GO at concentrations more than $50 \mathrm{mg} / \mathrm{L}$. Loss-of-function mutation of $\mathrm{n} / \mathrm{g}-1$ encoding a neuroligin with the function in connecting preand post-synaptic neurons suppressed the aversive behavior of nematodes to GO. Moreover, based on the neuronspecific activity assay, we found that the NLG-1 activity in AIY or AIB interneurons was required for the regulation of aversive behavior to GO. The neuron-specific activities of NLG-1 in AIY or AIB interneurons were also required for the regulation of $\mathrm{GO}$ toxicity.
\end{abstract}

Conclusions: Using nlg-1 mutant as a genetic tool, we identified the AIY and AIB interneurons required for the regulation of aversive behavior to GO. Our results provide an important neuronal basis for the aversive response of animals to environmental nanomaterials.

Keywords: Graphene oxide, NLG-1/neuroligin, Aversive behavior, Interneurons, Caenorhabditis elegans

\section{Background}

With the rapid increase in nanotechnology, a larger amount of engineered nanomaterials (ENMs) have been generated for industrial and medical applications. Among these ENMs, graphene nanomaterials have attracted massive attention due to their unique mechanical, electronic, and thermal properties [1,2]. Graphene oxide (GO) is a member of graphene nanomaterials, and can be potentially used in biomedicine, biosensor, and environmental remediation [3-6]. Meanwhile, some evidence from in vivo studies in mammals has demonstrated the potential of GO in inducing pulmonary or reproductive toxicity [7-9]. More recently, it has been further demonstrated that GO could cause the neurotoxicity on

\footnotetext{
*Correspondence: dayongw@seu.edu.cn

${ }^{2}$ Key Laboratory of Environmental Medicine Engineering in Ministry of Education, Medical School, Southeast University, Nanjing 210009, China

Full list of author information is available at the end of the article
}

zebrafish [10]. In contrast, how the animals evade the GO particles is still largely unclear.

The classic model animal of Caenorhabditis elegans has already been widely used in the toxicological study $[11,12]$. Using C. elegans as an in vivo assay system, it has been shown that GO could result in toxicity on the functions of both primary targeted organs, such as intestine, and secondary targeted organs, such as reproductive organs [13-17]. C. elegans is also a wonderful animal model for the study of neurotoxicity of certain toxicants $[11,18,19]$. In C. elegans, GO exposure has also been found to be neurotoxic for animals [20,21]. Additionally, neuronal ERK- or neuroligin/NLG-1-mediated molecular signaling regulated the formation of GO toxicity in nematodes [22, 23].

In organisms, the postsynaptic cell adhesion proteins, such as the neuroligins, act as central organizing molecules to connect pre- and post-synaptic neurons by binding to presynaptic proteins, like the neurexins [24,

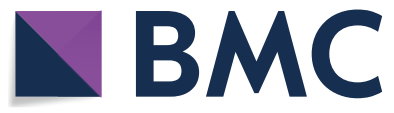

(c) The Author(s) 2018. This article is distributed under the terms of the Creative Commons Attribution 4.0 International License (http://creativecommons.org/licenses/by/4.0/), which permits unrestricted use, distribution, and reproduction in any medium, provided you give appropriate credit to the original author(s) and the source, provide a link to the Creative Commons license, and indicate if changes were made. The Creative Commons Public Domain Dedication waiver (http://creativecommons.org/ publicdomain/zero/1.0/) applies to the data made available in this article, unless otherwise stated. 
25]. In C. elegans, the single neuroligin gene is $n l g-1$. In this study, we first investigated the aversive behavior of nematodes to GO. Interneurons (also called connector neurons) establish the link between sensory neurons and motor neurons to enable the neuronal communication $[26,27]$. Moreover, using $n l g-1$ mutant as a genetic tool, we identified the interneurons required for the response of nematodes to GO exposure. Our data suggest the crucial role of AIY and AIB interneurons in the regulation of aversive response of nematodes to GO. Our results provide an important basis for the further elucidation of neuronal circuit for the response of nematodes to GO exposure.

\section{Methods}

\section{Preparation and characterization of GO}

GO was prepared from natural graphite powder based on the modified Hummer's method [28]. GO was finally obtained by ultrasonication of the as-made graphite oxide. Based on analysis of atomic force microscopy (AFM, SPM-9600, Shimadzu, Japan), the thickness of GO was approximately $1.0 \mathrm{~nm}$ in the topographic height, corresponding to one layer property (Fig. 1a). After sonication $(40 \mathrm{kHz}, 100 \mathrm{~W}, 30 \mathrm{~min})$, sizes of most of the GO were in the range of 40-50 $\mathrm{nm}$ based on the analysis of Nano Zetasizer (Malvern Instrument Ltd., Malvern, UK) (Fig. 1a, b). GO showed the typical G band and D band in Raman spectroscopy [29]. The zeta potential of GO $(100 \mathrm{mg} / \mathrm{L})$ in K-medium was $-21.5 \pm 2.6 \mathrm{mV}$ [29].

\section{C. elegans strains}

The used nematode strains were wild-type N2, mutants of $n \lg -1$ (tm474) and $n l g-1$ (ok259), and transgenic strains of $n l g-1($ ok259)Ex(Pttx-3-nlg-1),
nlg-1(ok259)Ex(Pgcy-28.d-nlg-1), nlg-1(ok259)Ex(Pnpr9-nlg-1), and nlg-1(ok259)Ex(Punc-86-nlg-1). Both nlg1 (tm474) and $n l g-1(o k 259)$ are loss-of-function mutants. Some of the strains were obtained from Caenorhabditis Genetics Center. Gravid hermaphrodite nematodes were maintained on normal nematode growth medium (NGM) plates seeded with Escherichia coli OP50 at $20{ }^{\circ} \mathrm{C}$ as described [30]. The gravid hermaphrodite nematodes were lysed with a bleaching mixture $(0.45 \mathrm{M} \mathrm{NaOH}, 2 \%$ $\mathrm{HOCl}$ ) in order to separate the eggs and the animals. Age synchronous L1-larvae or L4-larvae populations were prepared as described [31].

\section{Aversive response to $\mathrm{GO}$}

$\mathrm{GO}$ at the used working concentrations (50, 100, and $200 \mathrm{mg} / \mathrm{L})$ was prepared by diluting stock solution $(1 \mathrm{mg} /$ $\mathrm{mL}$ ) with $\mathrm{K}$ medium. Before the treatment, GO solutions were sonicated for $30 \mathrm{~min}(40 \mathrm{kHz}, 100 \mathrm{~W})$. To evaluate the aversive responses of nematodes to GO, half of the surface of a $6 \mathrm{~cm}$ diameter assay NGM plate was added with GO solution at different concentrations (region A). And then, the examined L4-larvae stage nematodes were placed at the center of the assay NGM plate. After 90 min treatment, the animals on the region $\mathrm{A}$ and on the opposite side (region B) were counted, respectively (Fig. 2a). The animals in the middle of the surface of assay NGM plate were omitted. The aversive response of nematodes to $\mathrm{GO}$ was evaluated by the percentage of $\mathrm{A} /(\mathrm{A}+\mathrm{B})$ (Fig. 2a). Forty nematodes were examined per treatment, and ten replicates were performed.

\section{Toxicity assessment of GO}

In nematodes, prolonged exposure (from L1-larvae to young adults) to $\mathrm{GO}$ at concentrations more than
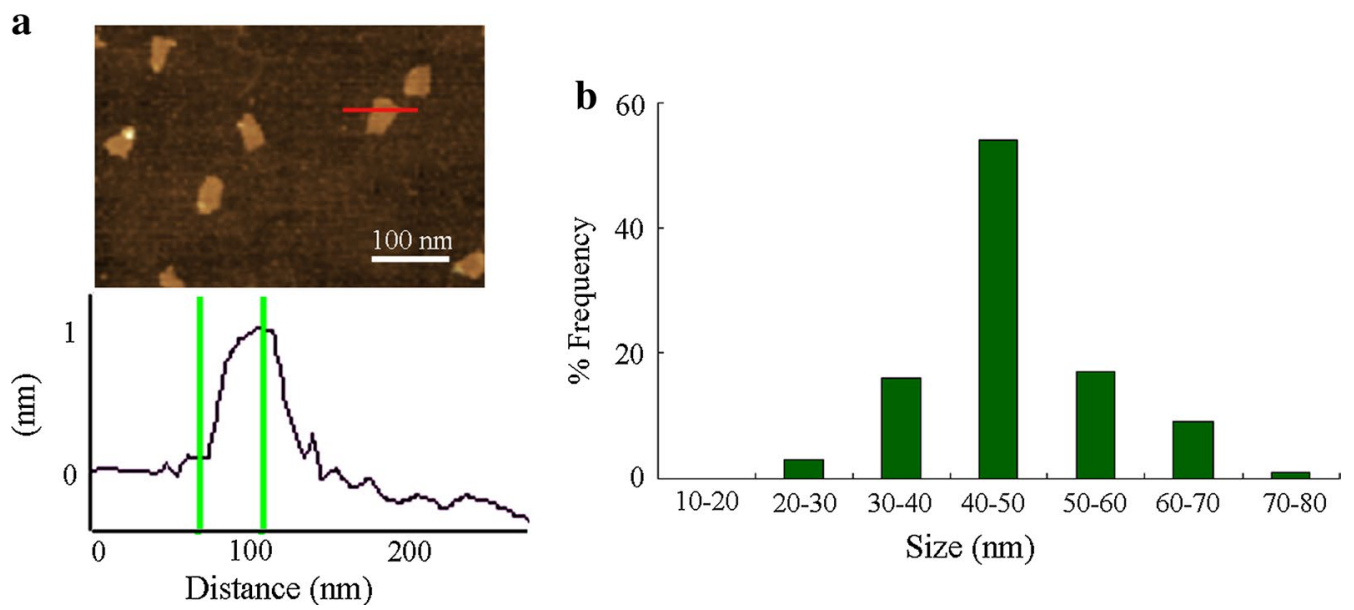

Fig. 1 Physiochemical properties of GO. a AFM analysis of GO. b Size distribution of GO after sonication based on the analysis of Nano Zetasizer 
$\mathbf{a}$

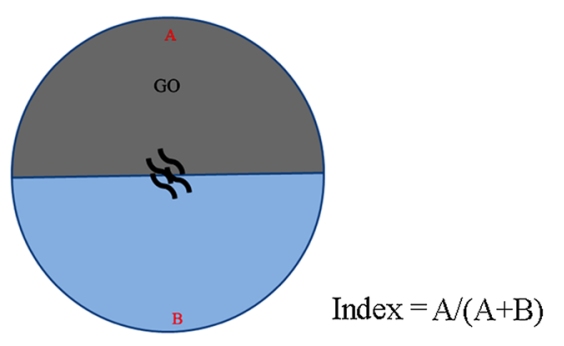

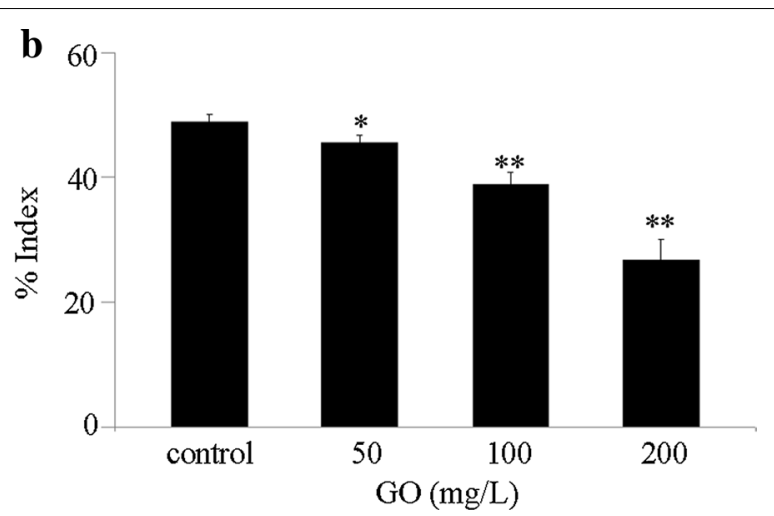

Fig. 2 Aversive behavior of wild-type nematodes to GO. a Assay model for aversive behavior of nematodes to GO. b Aversive behavior of wild-type nematodes to $\mathrm{GO}$ at different concentrations. Control, without GO treatment. Bars represent mean \pm SD. ${ }^{*} P<0.05$ vs control, ${ }^{* *} P<0.01$ vs control

$0.5 \mathrm{mg} / \mathrm{L}$ could cause the decrease in locomotion behavior and the induction of intestinal reactive oxygen species (ROS) production [14]. The used working solution $(10 \mathrm{mg} / \mathrm{L})$ was prepaed by diluting stock solution $(1 \mathrm{mg} /$ $\mathrm{mL}$ ) with $\mathrm{K}$ medium. Before the exposure, GO solution was sonicated for $30 \mathrm{~min}(40 \mathrm{kHz}, 100 \mathrm{~W})$. Prolonged exposure to $\mathrm{GO}$ was performed from L1-larvae to young adults in 12-well sterile tissue culture plates at $20{ }^{\circ} \mathrm{C}$ in the presence of food (OP50). After prolonged exposure, the GO exposed nematodes were used for the toxicity assessment using intestinal ROS production and locomotion behavior as the endpoints.

Intestinal ROS production can be used to reflect the functional state of intestine [32]. ROS production was analyzed as described previously [33, 34]. The nematodes were transferred to $1 \mu \mathrm{M} 5^{\prime}, 6^{\prime}$-chloromethyl-2', $7^{\prime}$ dichlorodihydro-fluorescein diacetate (CM-H2DCFDA) to incubate for $3 \mathrm{~h}$ at $20{ }^{\circ} \mathrm{C}$ in the dark. The examined nematodes were examined at $488 \mathrm{~nm}$ of excitation wavelength and $510 \mathrm{~nm}$ of emission filter under a laser scanning confocal microscope (Leica, TCS SP2, Bensheim, Germany). Relative fluorescence intensity in intestine was semi-quantified, and the semi-quantified ROS was expressed as relative fluorescence units (RFU) and normalized to the autofluorescence. Fifty nematodes were examined per treatment.

Head thrash and body bend were used to reflect the locomotion behavior [35]. These endpoints were analyzed under dissecting microscope as described [36, 37]. A head thrash is defined as a change in the direction of bending at the mid body, and a body bend is defined as a change in the direction of the part of the nematodes corresponding to the posterior bulb of the pharynx along the $y$ axis, assuming that nematode was traveling along the $x$ axis. Fifty nematodes were examined per treatment.
DNA constructs and germline transformation

Promoter region for $t t x-3$ gene specially expressed in AIY interneurons, $g c y$-28.d gene specially expressed in AIA interneurons, $n p r-9$ gene specially expressed in AIB interneurons, or unc-86 gene expressed in AIZ interneurons, was amplified by PCR from wild-type C. elegans genomic DNA. These promoter fragments were inserted into pPD95_77 vector in the sense orientation. nlg-1/ C40C9.5e cDNA was amplified by polymerase chain reaction (PCR), and inserted into corresponding entry vector carrying the $t t x-3, g c y-28 . d$, npr-9, or unc-86 promoter sequence. Germline transformation was performed as described by coinjecting testing DNA at the concentration of $10-40 \mu \mathrm{g} / \mathrm{mL}$ and marker DNA of Pdop-1::rfp at the concentration of $60 \mu \mathrm{g} / \mathrm{mL}$ into the gonad of nematodes [38]. The related primer information for DNA constructs is shown in Additional file 1: Table S1.

\section{Statistical analysis}

Data in this article were expressed as mean \pm standard deviation (SD). Statistical analysis was performed using SPSS 12.0 software (SPSS Inc., Chicago, USA). Differences between groups were determined using analysis of variance (ANOVA), and probability levels of 0.05 and 0.01 were considered statistically significant.

\section{Results}

Aversive behavior of wild-type nematodes to GO

On normal NGM plates without the addition of GO, the wild-type nematodes will run randomly, and would be distributed equally on the surface of NGM plates (Fig. 2b). In the aversive behavior assay model, we observed the significant aversive behavior of wild-type nematodes to GO at concentrations of 100 or $200 \mathrm{mg} / \mathrm{L}$ after 90 min treatment (Fig. 2b). We also detected the moderate but significant aversive behavior of wild-type 
nematodes to $\mathrm{GO}$ at the concentration of $50 \mathrm{mg} / \mathrm{L}$ after 90 min treatment (Fig. 2b). In contrast, after 90 min treatment, we did not observe the obvious aversive behavior of wild-type nematodes to $\mathrm{GO}$ at concentrations less than $50 \mathrm{mg} / \mathrm{L}$ (data not shown).

\section{nlg-1 mutation suppressed the aversive behavior of nematodes to $\mathrm{GO}$}

Considering the important function of neuroligins in connecting pre- and post-synaptic neurons [24, 25], we next examined the effect of $n l g-1$ mutation on aversive behavior of nematodes to GO. We focused on the analysis of aversive behavior of nematodes to GO at the concentration of $100 \mathrm{mg} / \mathrm{L}$ (Fig. 3a). On normal NGM plates, both wild-type and $n l g-1$ mutant (nlg-1(ok259) or $n \lg -1(\operatorname{tm} 474))$ nematodes were observed to be distributed equally on the surface of NGM plates (Fig. 3b).
In the aversive behavior assay model, both $n \lg -1$ (ok259) mutant and $n l g-1(t m 474)$ mutant showed the increased index for assessing aversive behavior to GO $(100 \mathrm{mg} / \mathrm{L})$ compared with wild-type nematodes after 90 min treatment (Fig. 3b). Therefore, $n l g-1$ mutation may suppress the aversive behavior of nematodes to GO in nematodes.

\section{Neuron-specific activity of NLG-1 in the regulation of aversive behavior of nematodes to $\mathrm{GO}$}

In C. elegans, AIY, AIA, AIB, and AIZ interneurons are main classes of integrating neurons between sensory neurons and motor neurons (Fig. 4a) [39]. After $90 \mathrm{~min}$ treatment, we found that expression of $n l g-1$ in AIA interneurons or AIZ interneurons could not rescue the deficit in aversive behavior to GO $(100 \mathrm{mg} / \mathrm{L})$ in $n l g$ 1(ok259) mutant nematodes (Fig. 4b). In contrast, after 90 min treatment, neuron-specific expression of $n l g-1$ in
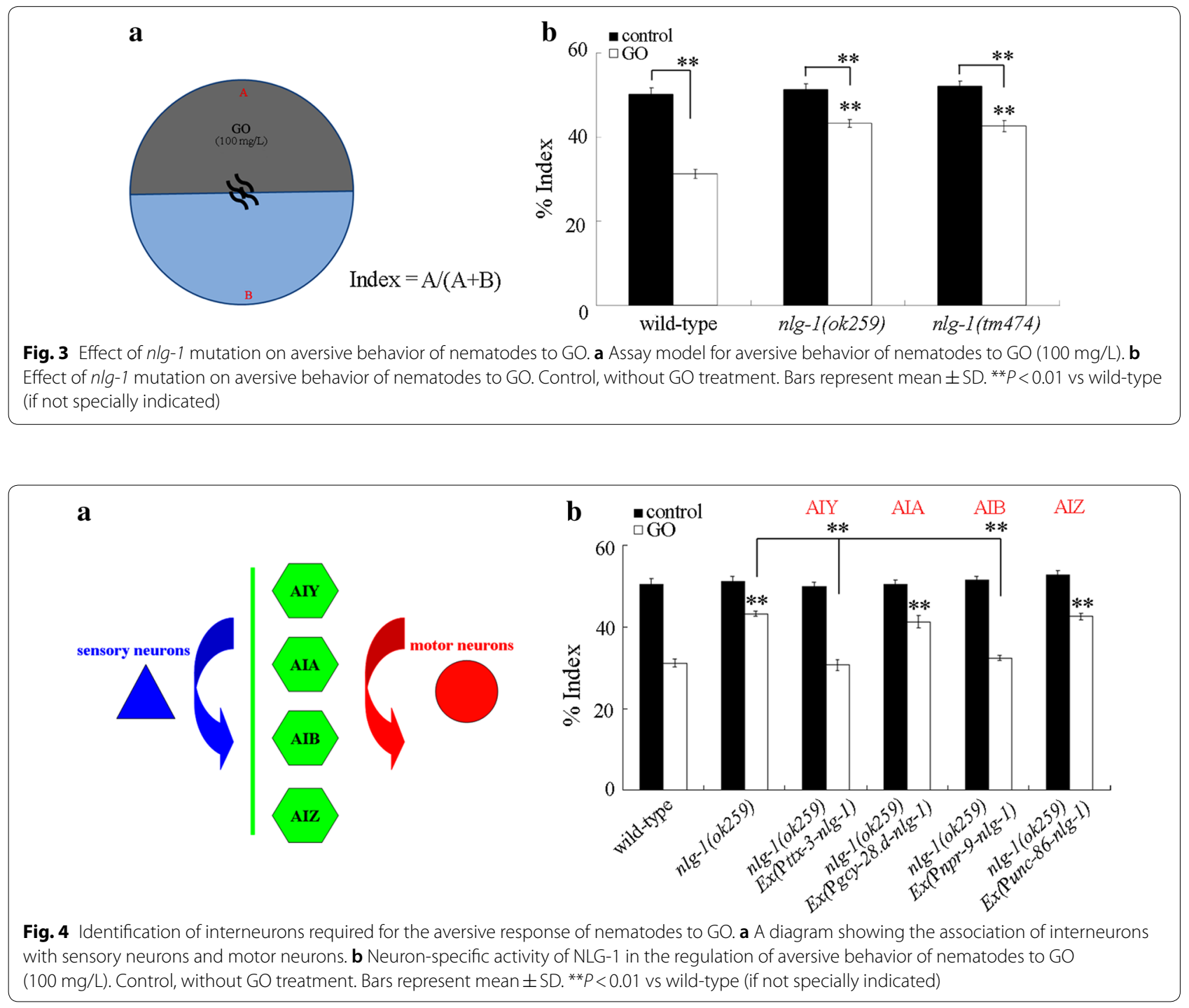
AIY interneurons or AIB interneurons could significantly decrease the index of aversive behavior to GO $(100 \mathrm{mg} / \mathrm{L})$ in $n l g-1$ (ok259) mutant nematodes (Fig. 4b). Therefore, neuron-specific expression of $n l g-1$ in AIY interneurons or AIB interneurons can rescue the deficit in aversive behavior to GO in $n l g-1$ (ok259) mutant nematodes.

\section{Neuron-specific activity of NLG-1 in the regulation of GO toxicity}

We further determined the roles of these four classes of interneurons in the regulation of GO toxicity. Using intestinal ROS production and locomotion behavior as the toxicity assessment endpoints, we observed that expression of $n l g-1$ in AIA interneurons or AIZ interneurons could not obviously affect the GO toxicity in inducing intestinal ROS production and in decreasing locomotion behavior in $n l g-1$ (ok259) mutant nematodes (Fig. 5). In contrast, expression of $n l g-1$ in AIY interneurons or AIB interneurons significantly suppress the GO toxicity in inducing intestinal ROS production and in decreasing locomotion behavior in $n l g-1$ (ok259) mutant nematodes (Fig. 5). Therefore, both AIY interneurons and AIB interneurons are also required for the regulation of GO toxicity in nematodes.

\section{Discussion}

Caenorhabditis elegans is a useful model for toxicity assessment of environmental toxicants [11, 12]. In this study, using the in vivo assay system of $C$. elegans, we observed the obvious aversive behavior of

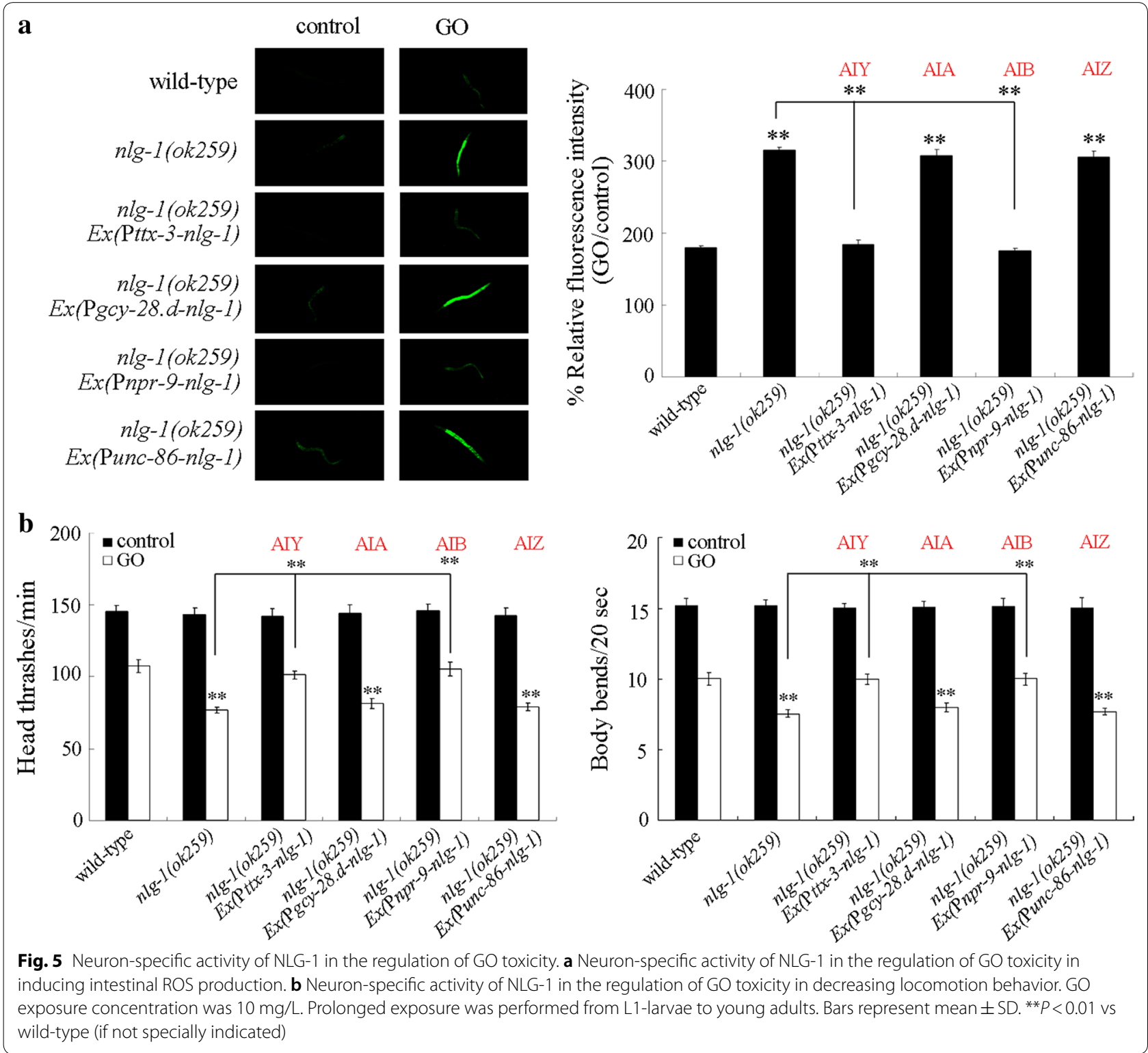


nematodes to GO particles (Fig. 2). Moreover, we also observed the significant aversive behavior of nematodes to $\mathrm{TiO}_{2}$-nanoparticles $\left(\mathrm{TiO}_{2}-\mathrm{NPs}, 10 \mathrm{~nm}\right), \mathrm{Al}_{2} \mathrm{O}_{3}-\mathrm{NPs}$ (60 nm), multi-walled carbon nanotubes (MWCNTs), or thiolated GO (GO-SH) in nematodes (Additional file 1: Fig. S1). The detailed information on the physicochemical properties of examined $\mathrm{TiO}_{2}-\mathrm{NPs}, \mathrm{Al}_{2} \mathrm{O}_{3}-\mathrm{NPs}, \mathrm{MWC}$ NTs, or GO-SH is available in the references [40-43]. These observations imply that the nematodes have the potential ability to avoid the environmental ENMs once percept the existence of ENMs in the environment. This observed aversive response enables the environmental animals a protection mechanism to reduce the possible toxicity of environmental ENMs. Nevertheless, the examined GO at environmentally relevant concentrations may not be able to induce the aversive behavior of nematodes after 90 min treatment.

In C. elegans, it has been shown that NLG-1/neuroligin is required for the control of synaptic function, a subset of sensory behaviors and sensory processing, longevity, and oxidative stress or stress response [23, 44-47]. In this study, we further found a novel function of NLG-1 in the regulation of aversive behavior to GO. Loss-of-function mutation of $n l g-1$ significantly suppress the aversive behavior to GO (Fig. 3), implying that NLG-1/neuroligin is required for the formation of normal aversive behavior to GO. Because the neuroligins act as a link to connect pre- and post-synaptic neurons in organisms [24, 25], our results suggest that a certain neuronal circuit connected by NLG-1/neuroligins may exist to regulate the aversive behavior of nematodes to GO.

We further provide the evidence to demonstrate the crucial function of interneurons in the regulation of aversive behavior to GO. Among the main classes of interneurons with the function to integrate sensory neurons with motor neurons [39], we observed that only expression of $n l g-1$ in AIY interneurons or AIB interneurons could recover the deficits in aversive behavior to GO in $n l g-1$ mutant nematodes (Fig. 4). In contrast, the neuron-specific activity of $n l g-1$ in AIA or AIZ interneurons was not required for the function of NLG-1 in the regulation of aversive behavior to GO (Fig. 4). These results imply that AIY and AIB interneurons are involved in the regulation of aversive behavior to GO (Fig. 6). Because the integration between sensory neurons and motor neurons by interneurons is conserved between the nematodes and the mammals or the human, our results further imply the crucial role of interneurons in the perception of toxic ENMs in mammals or in human.

In C. elegans, genetic or laser ablation of AIY interneurons caused the abnormal spontaneous reversal rate, odorant chemotaxis, and salt chemotaxis [48-50]. Additionally, genetic or laser ablation of AIB interneurons

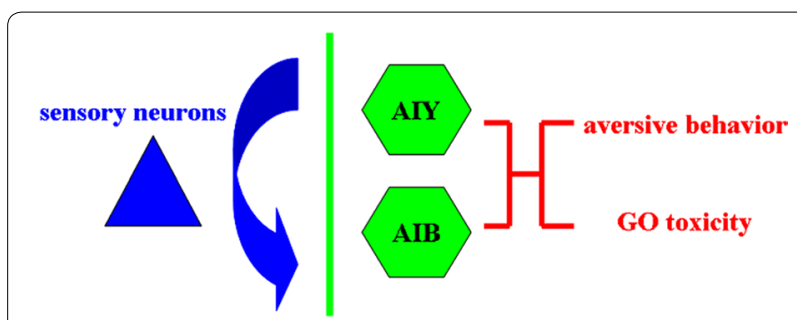

Fig. 6 A diagram showing the functions of AlY and AIB interneurons in the regulation of aversive behavior to $\mathrm{GO}$ and $\mathrm{GO}$ toxicity

caused the abnormal odorant chemotaxis, and salt chemotaxis $[49,50]$. Our results imply the novel function of AIY and AIB interneurons in the control of aversive behavior of nematodes to environmental toxicants. In $C$. elegans, it was repotted that genetic or laser ablation of AIY interneurons can enhance the spontaneous reversal rate [48]. Therefore, the identified interneurons may not only mediate a certain neuronal circuit to regulate the aversive behavior to GO, but also be able to directly participate in the regulation of aversive behavior themselves.

Our previous study has identified the neuron-specific activity of $n l g-1$ in the AIY interneurons in the regulation of GO toxicity [23]. In this study, we further found the neuron-specific activity of $n l g-1$ in the AIB interneurons in the regulation of GO toxicity (Fig. 5). These results all imply the crucial function of NLG-1 in interneurons in the regulation of GO toxicity (Fig. 6). In C. elegans, AIY interneurons act as the output of ASE, AWC, AFD, and AWA sensory neurons, and AIB interneurons act as the output of ASE, AWC, ASI, ASH, ASK, ADL, AFD, and ASG sensory neurons [38]. In C. elegans, the neurexin gene is $n r x-1$. The further examination of neuron-specific activities of NRX-1 will be helpful for final identification of neuronal circuit required for the control of aversive behavior to $\mathrm{GO}$ in nematodes.

\section{Conclusions}

In this study, we investigated the aversive response of animals to GO using the in vivo assay system of C. elegans. We observed the obvious aversive behavior of nematodes to $\mathrm{GO}$ at concentrations more than $50 \mathrm{mg} / \mathrm{L}$. In nematodes, mutation of $n l g-1$ encoding a neuroligin disrupted this aversive behavior to GO. Using $n l g-1$ mutant as a genetic tool, we identified the AIY and AIB interneurons to be required for the regulation of aversive behavior to GO based on a series of rescue assays. Our results provide the important neuronal and molecular basis for the aversive response of animals to GO. Moreover, we found that both the AIY interneurons and the AIB interneurons were also required for the regulation of $\mathrm{GO}$ toxicity in nematodes. 


\section{Additional file}

Additional file 1. Additional table and figure.

\section{Authors' contributions}

Experiments were designed by DW and conducted by GX, HC, NK, and QL. Data was analyzed by GX and HC. Manuscript was prepared by DW and edited by DW. All authors read and approved the final manuscript.

\begin{abstract}
Author details
${ }^{1}$ College of Biology and Food Engineering, Chongqing Three Gorges University, Wanzhou 404100, China. ${ }^{2}$ Key Laboratory of Environmental Medicine Engineering in Ministry of Education, Medical School, Southeast University, Nanjing 210009, China. ${ }^{3}$ Institute of Biophysics and Biomedical Engineering, Bulgarian Academy of Science, Sofia 1113, Bulgaria. ${ }^{4}$ School of Public Health, Nanjing Medical University, Nanjing 211166, China.
\end{abstract}

\section{Acknowledgements}

Not applicable.

\section{Competing interests}

The authors declare that they have no competing interests.

\section{Availability of data and materials \\ All the data and materials are available.}

\section{Consent for publication}

All the co-authors were aware of this submission and approve for publication.

\section{Ethics approval and consent to participate}

This study was performed in accordance with the Guide for the Care and Use of Laboratory Animals and was approved by the Committee of Ethics of Animal Experimentation of the Southeast University.

\section{Funding}

This work was supported by the Grants from Joint Project between Southeast University and Nanjing Medical University (No. 2242017K3DN07), and Bilateral Projects 2016 China-Bulgaria (No. 15-4).

\section{Publisher's Note}

Springer Nature remains neutral with regard to jurisdictional claims in published maps and institutional affiliations.

Received: 17 September 2017 Accepted: 23 April 2018

Published online: 27 April 2018

\section{References}

1. Geim AK. Graphene: status and prospects. Science. 2009;324:1530-4.

2. Qiu J, et al. Effects of graphene quantum dots on the self-renewal and differentiation of mesenchymal stem cells. Adv Healthc Mater. 2016;5:702-10.

3. Geim AK, Novoselov KS. The rise of graphene. Nat Mater. 2007;6:183-91.

4. Bitounis $D$, et al. Prospects and challenges of graphene in biomedical applications. Adv Mater. 2013;25:2258-68.

5. Zhu X, et al. Brianyoungite/graphene oxide coordination composites for high-performance $\mathrm{Cu}^{2+}$ adsorption and tunable deep-red photoluminescence. ACS Appl Mater Interfaces. 2016;8:15848-54.

6. Cheng C, Wang D. Hydrogel-assisted transfer of graphene oxides into nonpolar organic media for oil decontamination. Angew Chem Int Ed. 2016;55:6853-7.

7. Yang K, et al. Behavior and toxicity of graphene and its functionalized derivatives in biological systems. Small. 2013;9:1492-503.

8. Li B, et al. Biodistribution and pulmonary toxicity of intratracheally instilled graphene oxide in mice. NPG Asia Mater. 2013;5:e44.
9. Liang $\mathrm{S}$, et al. Reproductive toxicity of nanosclae graphene oxide in male mice. Biomaterials. 2015;9:92-105.

10. Soares JC, et al. Developmental neurotoxic effects of graphene oxide exposure in zebrafish larvae (Danio rerio). Colloids Surf B Biointerfaces. 2017;157:335-46.

11. Leung MC, et al. Caenorhabditis elegans: an emerging model in biomedical and environmental toxicology. Toxicol Sci. 2008;106:5-28.

12. Zhao Y-L, et al. Translocation, transfer, and in vivo safety evaluation of engineered nanomaterials in the non-mammalian alternative toxicity assay model of nematode Caenorhabditis elegans. RSC Adv. 2013;3:5741-57.

13. Zhang W, et al. Unraveling stress-induced toxicity properties of graphene oxide and the underlying mechanism. Adv Mater. 2012;24:5391-7.

14. Wu Q-L, et al. Contributions of altered permeability of intestinal barrier and defecation behavior to toxicity formation from graphene oxide in nematode Caenorhabditis elegans. Nanoscale. 2013;5:9934-43.

15. Wu Q-L, et al. Genome-wide identification and functional analysis of long noncoding RNAs involved in the response to graphene oxide. Biomaterials. 2016;102:277-91.

16. Zhao Y-L, et al. Glycyrrhizic acid, active component from Glycyrrhizae radix, prevents toxicity of graphene oxide by influencing functions of microRNAs in nematode Caenorhabditis elegans. Nanomedicine. 2016;12:735-44.

17. Zhi L-T, et al. Wnt ligands differentially regulate toxicity and translocation of graphene oxide through different mechanisms in Caenorhabditis elegans. Sci Rep. 2016;6:39261.

18. Yu X-M, et al. Vitamin E ameliorates the neurodegeneration related phenotypes caused by neurotoxicity of $\mathrm{Al}_{2} \mathrm{O}_{3}$-nanoparticles in C. elegans. Toxicol Res. 2015:4:1269-81.

19. Zhao Y-L, et al. Quantum dots exposure alters both development and function of D-type GABAergic motor neurons in nematode Caenorhabditis elegans. Toxicol Res. 2015;4:399-408.

20. Li P, et al. Chronic exposure to graphene-based nanomaterials induces behavioral deficits and neural damage in Caenorhabditis elegans. J Appl Toxicol. 2017;37:1140-50.

21. Zhi L-T, et al. Graphene oxide induces canonical Wnt/ $\beta$-catenin signalingdependent toxicity in Caenorhabditis elegans. Carbon. 2017;113:122-31.

22. Qu M, et al. Neuronal ERK signaling in response to graphene oxide in nematode Caenorhabditis elegans. Nanotoxicology. 2017;11:520-33.

23. Chen H, Li H-R, Wang D-Y. Graphene oxide dysregulates neuroligin/ NLG-1-mediated molecular signaling in interneurons in Caenorhabditis elegans. Sci Rep. 2017:7:41655.

24. Südhof TC. Neuroligins and neurexins link synaptic function to cognitive disease. Nature. 2008:455:903-11.

25. Missler M, Südhof TC, Biederer T. Synaptic cell adhesion. Cold Spring Harb Perspect Biol. 2012;4:a005694.

26. Whittington MA, et al. Inhibition-based rhythms: experimental and mathematical observations on network dynamics. Int J Psychophysiol. 2000:38:315-36.

27. Kandel E, Schwartz J, Jessell T. Principles of neural science. 4th ed. New York City: McGraw Hill Companies; 2000.

28. Kovtyukhova NI, et al. Layer-by-layer assembly of ultrathin composite films from micron-sized graphite oxide sheets and polycations. Chem Mater. 1999;11:771-8.

29. Yang R-L, et al. A mir-231-regulated protection mechanism against the toxicity of graphene oxide in nematode Caenorhabditis elegans. Sci Rep. 2016;6:32214.

30. Brenner S. The genetics of Caenorhabditis elegans. Genetics. 1974;77:71-94.

31. Donkin S, Williams PL. Influence of developmental stage, salts and food presence on various end points using Caenorhabditis elegans for aquatic toxicity testing. Environ Toxicol Chem. 1995;14:2139-47.

32. Zhao Y-L, et al. p38 MAPK-SKN-1/Nrf signaling cascade is required for intestinal barrier against graphene oxide toxicity in Caenorhabditis elegans. Nanotoxicology. 2016;10:1469-79.

33. Wu Q-L, et al. Inhibition of ROS elevation and damage on mitochondrial function prevents lead-induced neurotoxic effects on structures and functions of AFD neurons in Caenorhabditis elegans. J Environ Sci. 2012;24:733-42.

34. Yang J-N, et al. Toxicity evaluation and translocation of carboxyl functionalized graphene in Caenorhabditis elegans. Toxicol Res. 2015;4:1498-510. 
35. Zhao Y-L, Yang J-N, Wang D-Y. A microRNA-mediated insulin signaling pathway regulates the toxicity of multi-walled carbon nanotubes in nematode Caenorhabditis elegans. Sci Rep. 2016;6:23234.

36. Liu Z-F, et al. Crucial role of intestinal barrier in the formation of transgenerational toxicity in quantum dots exposed nematodes Caenorhabditis elegans. RSC Adv. 2015;5:94257-66.

37. Ren $M-X$, et al. Antimicrobial proteins in the response to graphene oxide in Caenorhabditis elegans. Nanotoxicology. 2017;11:578-90.

38. Mello C, Fire A. DNA transformation. Methods Cell Biol. 1995:48:451-82.

39. White JG, et al. The structure of the nervous system of the nematode Caenorhabditis elegans. Philos Tran R Soc B Biol Sci. 1986;314:1-340.

40. Wu Q-L, et al. Susceptible genes regulate the adverse effects of $\mathrm{TiO}_{2}-\mathrm{NPs}$ at predicted environmental relevant concentrations on nematode Caenorhabditis elegans. Nanomedicine. 2014;10:1263-71.

41. Li Y-X, et al. Transmissions of serotonin, dopamine and glutamate are required for the formation of neurotoxicity from $\mathrm{Al}_{2} \mathrm{O}_{3}-\mathrm{NPs}$ in nematode Caenorhabditis elegans. Nanotoxicology. 2013;7:1004-13.

42. Zhao $L$, et al. Multi-walled carbon nanotubes-induced alterations in microRNA let-7 and its targets activate a protection mechanism by conferring a developmental timing control. Part Fibre Toxicol. 2017:14:27.

43. Ding $X-C$, et al. Long-term exposure to thiolated graphene oxide in the range of $\mu \mathrm{g} / \mathrm{L}$ induces toxicity in nematode Caenorhabditis elegans. Sci Total Environ. 2018;616-617:29-37.
44. Shen $L-L$, Wang $Y$, Wang $D-Y$. Involvement of genes required for synaptic function in aging control in C. elegans. Neurosci Bull. 2007;23:21-9.

45. Hunter JW, et al. Neuroligin-deficient mutants of $C$. elegans have sensory processing deficits and are hypersensitive to oxidative stress and mercury toxicity. Dis Model Mech. 2010;3:366-76.

46. $\mathrm{Hu} \mathrm{Z}$, et al. Neurexin and neuroligin mediate retrograde synaptic inhibition in C. elegans. Science. 2012;337:980-4.

47. Staab TA, et al. Regulation of synaptic $n / g-1 /$ neuroligin abundance by the skn-1/Nrf stress response pathway protects against oxidative stress. PLoS Genet. 2014;10:e1004100.

48. Tsalik EL, Hobert O. Functional mapping of neurons that control locomotory behavior in Caenorhabditis elegans. J Neurobiol. 2003;56:178-97.

49. lino Y, Yoshida K. Parallel use of two behavioral mechanisms for chemotaxis in Caenorhabditis elegans. J Neurosci. 2009;29:5370-80.

50. Taniguchi $\mathrm{G}$, et al. Screening of odor-receptor pairs in Caenorhabditis elegans reveals different receptors for high and low odor concentrations. Sci Signal. 2014;7:ra39.
Ready to submit your research? Choose BMC and benefit from:

- fast, convenient online submission

- thorough peer review by experienced researchers in your field

- rapid publication on acceptance

- support for research data, including large and complex data types

- gold Open Access which fosters wider collaboration and increased citations

- maximum visibility for your research: over 100M website views per year

At BMC, research is always in progress.

Learn more biomedcentral.com/submissions 